\title{
Mieloma múltiplo: características clínicas e laboratoriais ao diagnóstico e estudo prognóstico
}

\author{
Multiple myeloma: clinical and laboratory characteristics in the diagnosis and prognostic study
}

Roberta O. Paula e Silva ${ }^{1}$

Kamilla M. A. Brandão ${ }^{1}$

Paula V. M. Pinto ${ }^{1}$

Rosa M. D. Faria ${ }^{2}$

Nelma C. D. Clementino ${ }^{3}$

Cristiane M. F. Silva

Aline F. Lopes ${ }^{5}$
O mieloma múltiplo (MM) caracteriza-se por expansão clonal plasmocitária na medula óssea e produção de imunoglobulina monoclonal, promovendo progressivamente destruição óssea, falência renal, supressão da hematopoiética e infecções. A identificação de fatores clínicos e laboratoriais ao diagnóstico é importante para predizer sobrevida. $O$ sistema de estadiamento de Durie e Salmon (1975) é o mais utilizado e baseia-se na correlação entre parâmetros clínico-laboratoriais à massa tumoral. A combinação de ß2 microglobulina e albumina sérica resultou em um sistema de estadiamento simples e confiável, conhecido como Sistema Internacional de Estadiamento (ISS), que tem sido reconhecido atualmente. O objetivo deste trabalho foi analisar as características clínicas e laboratoriais, ao diagnóstico, de pacientes portadores de MM e estudo de sobrevida. Realizou-se estudo de coorte não concorrente de 101 pacientes portadores de MM atendidos no Serviço de Hematologia do Hospital das Clínicas da UFMG, que receberam diagnóstico no período de abril de 1994 a 31 de outubro de 2006, através da coleta de dados contidos em prontuários médicos. Os pacientes foram acompanhados até maio de 2007. Foi feita análise descritiva das características ao diagnóstico e estudo de sobrevida, utilizando-se análise univariada pela técnica do produto-limite de Kaplan \& Meier e teste de Log-Rank para comparação das curvas; já na análise multivariada, utilizou-se regressão múltipla de Cox. A mediana de idade dos pacientes foi de 63 anos de idade, 47,5\% eram homens e 52,5\% mulheres, sendo 50,6\% brancos, 33,3\% negros e 16,1\% pardos. Manifestações clínicas mais comuns foram: dor óssea (83,2\%), fraqueza (70,3\%) e perda de peso (40,6\%). Radiografia de esqueleto mostrou alterações em 83,8\% dos casos. Em relação ao sistema de estadiamento proposto por Durie \& Salmon, 63 (62,4\%) pacientes encontravam-se em estádio III, 32 (31,7\%) em estádio II e apenas 6 (5,9\%) no estádio I ao diagnóstico. Utilizando a classificação do Sistema Internacional de Estadiamento (ISS) obtiveram-se 22 (30,1\%) pacientes no estádio III, 31 (42,5\%) no estádio II e 20 (27,4\%) no estádio I ao diagnóstico. Sobrevida global média foi de 66,52 meses, com mediana de seguimento de 20 meses. Na análise univariada foram significantes: creatinina $(p=0,006)$, hemoglobina $(p=0,001)$, estadiamento de Durie \& Salmon $(p=0,009)$ e ISS $(p=0,014)$; e na multivariada somente a hemoglobina $(p=0,012)$ correlacionou-se à sobrevida. A maior parte dos pacientes apresentava estádio clínico avançado ao diagnóstico com repercussão na sobrevida. Rev. Bras. Hematol. Hemoter. 2009;31(2):63-68.

Palavras-chave: Mieloma múltiplo; prognóstico; características clínicas e laboratoriais.

\footnotetext{
${ }^{1}$ Médica Residente do Hospital das Clínicas da UFMG, Belo Horizonte-MG.

${ }^{2}$ Professora da Faculdade de Medicina da UFMG e coordenadora do Atendimento ao Paciente portador de Doença Linfoproliferativa do Serviço de Hematologia do Hospital das Clínicas da UFMG.

${ }^{3}$ Professora Associada do Departamento de Propedêtica Complementar e coordenadora do Ambulatório de Doenças Mieloproliferativas Crônicas do Serviço de Hematologia do Hospital das Clínicas da UFMG.

${ }^{4}$ Médica Hematologista do Hospital de Doenças Infectocontagiosas Dr. Clementino Fraga-PB.

${ }^{5}$ Médica Residente do Hospital Pronto Socorro João XXIII-MG.
}

Serviço de Hematologia do Hospital das Clínicas da Universidade Federal de Minas Gerais, Belo Horizonte-MG.

Correspondência: Roberta Oliveira de Paula e Silva

Departamento de Propedêtica Complementar, Faculdade de Medicina - UFMG

Av. Prof. Alfredo Balena, 190, sala 6000 - Santa Efigênia

30130-100 - Belo Horizonte-MG - Brasil

Tel.: (55 31)3248-9774; (55 31)9737-6877; Fax: (55 31)3497-4314 - E-mail: betaops@gmail.com

Doi: $10.1590 / S 1516-84842009005000013$ 


\section{Introdução}

O mieloma múltiplo (MM) é uma neoplasia progressiva e incurável de células B, caracterizada pela proliferação desregulada e clonal de plasmócitos na medula óssea (MO), os quais produzem e secretam imunoglobulina (Ig) monoclonal ou fragmento dessa, chamada proteína M. ${ }^{1}$ As consequências fisiopatológicas do avanço da doença incluem: destruição óssea, falência renal, supressão da hematopoese e maior risco de infecções. ${ }^{2}$ Essa neoplasia é frequentemente precedida por Gamopatia Monoclonal de Significado Indeterminado (MGUS). ${ }^{3,4}$ Representa $1 \%$ de todas as neoplasias malignas, sendo a segunda neoplasia hematológica mais comum. ${ }^{5} \mathrm{O}$ aumento da incidência do MM nos últimos anos relaciona-se ao maior conhecimento da história natural da doença e sua patogênese, à melhora dos recursos laboratoriais, ao aumento da expectativa de vida mundial e à exposição crônica a agentes poluentes. ${ }^{6}$

Após a confirmação do diagnóstico de MM, o paciente deve ser avaliado quanto ao prognóstico. ${ }^{7} \mathrm{O}$ sistema de estadiamento clínico proposto por Durie \& Salmon (1975), amplamente utilizado, baseia-se na combinação de fatores (hemoglobina, cálcio sérico, componente monoclonal, acometimento ósseo e creatinina sérica) que se correlacionam à massa tumoral. Porém, recentes avanços na biologia e tratamento da doença mostram que esse sistema não é capaz de relacionar adequadamente a sobrevida global ao tempo livre de doença. ${ }^{8}$ Portanto, novos parâmetros têm sido desenvolvidos para uma melhor correlação clínica e estratificação de subgrupos com diferentes evoluções. A combinação de $\beta_{2}$ microglobulina e albumina sérica resultou em um sistema de estadiamento simples e confiável, possibilitando uma divisão em três estádios clínicos, denominado Sistema Internacional de Estadiamento (ISS), ${ }^{7}$ como mostra o Quadro 1.

Em países desenvolvidos, a apresentação clínica dos pacientes ao diagnóstico tem ocorrido em estágios mais precoces, aproximadamente $20 \%$ dos pacientes apresentam-se assintomáticos ao diagnóstico, evidenciando-se apenas elevação da proteína monoclonal sérica ou urinária (MGUS). ${ }^{1}$ Porém, um estudo realizado na América do Sul por Hungria et al., ${ }^{9}$ incluindo pacientes diagnosticados com MM entre 1998 e 2002, mostrou que a maioria dos pacientes apresentava doença em estádio avançado, ao diagnóstico.

\section{Casuística e Método}

Foi realizado um estudo de coorte não concorrente, com o objetivo de se levantarem características clínicas e laboratoriais dos pacientes portadores de MM ao diagnóstico, através de dados registrados no prontuário médico, correlacionando-os com a sobrevida dos pacientes. Foram estudados pacientes do ambulatório de Doenças Linfoproliferativas do Serviço de Hematologia do Hospital das Clínicas da UFMG (HC-UFMG) que receberam diagnóstico
Quadro 1. Sistema Internacional de Estadiamento (ISS)

\begin{tabular}{l}
$\begin{array}{l}\beta 2 \text { microglobulina sérica }<3,5 \mathrm{mg} / \mathrm{dL} \\
\text { Estádio I }\end{array}$ \\
$\begin{array}{l}\text { Estádio II } \\
\text { Estádio III } \\
\text { Nem I nem III* }\end{array}$ \\
$\begin{array}{l}\text { *Existem } 2 \text { categorias no estádio II: } \\
\beta 2 \text { microglobulina sérica }<3,5 \mathrm{mg} / \mathrm{dL} \text {, mas albumina sérica }<3,5 \mathrm{~g} / \mathrm{dl} \\
\beta 2 \text { microglobulina sérica }>3,5 \mathrm{mg} / \mathrm{dL} \text {, mas albumina sérica }>3,5 \mathrm{~g} / \mathrm{dl}\end{array}$ \\
\hline
\end{tabular}

de MM no período de abril de 1994 a 31 de outubro de 2006. Os pacientes foram acompanhados até maio de 2007. A seleção dos pacientes foi realizada a partir dos registros de mielograma realizados no próprio serviço por suspeita de MM. Foram identificados, a príncipio, 166 pacientes, sendo que 35 pacientes foram excluídos da amostra final por não se tratarem de MM. Dos 131 pacientes restantes, 11 foram excluídos por não disporem dos dados necessários ao diagnóstico no prontuário e 19 por não terem o prontuário localizado no Serviço de Arquivo Médico e Estatística (SAME) do HC- UFMG. A amostra final foi de 101 pacientes (97 com MM sintomático e 4 com MM assintomático).

Os dados coletados, na ocasião do diagnóstico, foram: idade, sexo, cor (branca, negra e parda), manifestações clínicas, parâmetros laboratoriais ao diagnóstico (hemoglobina, plaquetas, cálcio total, ácido úrico, desidrogenase láctica, uréia, creatinina, eletroforese de proteínas séricas e/ou urinária, tipo de imunoglobulina e $\aleph_{2}$ microglobulina); escala de lesões ósseas ao raio-X, ( 0 - osso normal, 1 - osteoporose, 2 - lesões osteolíticas, 3 - destruição esquelética); estadiamento clínico de Dürie e Salmon, ISS e sobrevida.

Todos os dados foram codificados e analisados pelo programa SPSS (Statistical Package for the Social Sciences). Características clínicas e laboratoriais, foram analisadas de forma descritiva. Na análise univariada, a probabilidade de sobrevida e as curvas de sobrevida foram determinadas pela técnica do produto-limite de Kaplan-Meier. ${ }^{10}$ As curvas determinadas foram comparadas utilizando-se o teste Log Rank. As variáveis qualitativas foram diretamente comparadas com a sobrevida. Nas variáveis quantitativas, pontos de corte foram determinados de acordo com a literatura ou, quando necessário, determinamos vários pontos de corte, para melhor avaliação. Após a identificação das variáveis significantes à análise univariada, as mesmas foram submetidas à análise multivariada, utilizando-se a regressão múltipla de Cox, ${ }^{11}$ para determinarmos quais possuíam valor prognóstico independente e para quantificarmos sua importância na sobrevida. Em todos os testes fixou-se em 0,05 o nível de significância.

\section{Resultados e Discussão}

A idade dos pacientes variou de 32 a 87 anos, com mediana de 63 anos de idade (DP=13,0). Estudo realizado por Kyle et $a .^{2}$ mostrou que a média de idade ao diagnóstico foi 
de 66 anos, enquanto estudo realizado no Brasil, mostrou média de idade, ao diagnóstico, de 61 anos. ${ }^{12}$ Em relação ao sexo, 48 (47,5\%) eram do sexo masculino e 53 (52,5\%) do sexo feminino. A maior prevalência em mulheres contrasta com dados da literatura, ${ }^{1,2}$ o que pode ser justificado pelo tamanho da amostra ou características próprias dessa população. Hungria et al., ${ }^{9}$ em estudo realizado em diversos centros brasileiros, mostraram $52 \%$ de prevalência do sexo feminino e $48 \%$ do masculino. Houve predomínio da cor branca -44 (50,6\%), seguida pela negra - 29 (33,3\%) e parda - 14 (16,1\%). Em 14 pacientes não foi possível identificação da cor através dos dados obtidos nos prontuários. Apesar da literatura demonstrar maior prevalência em negros, ${ }^{13,14}$ alguns estu-

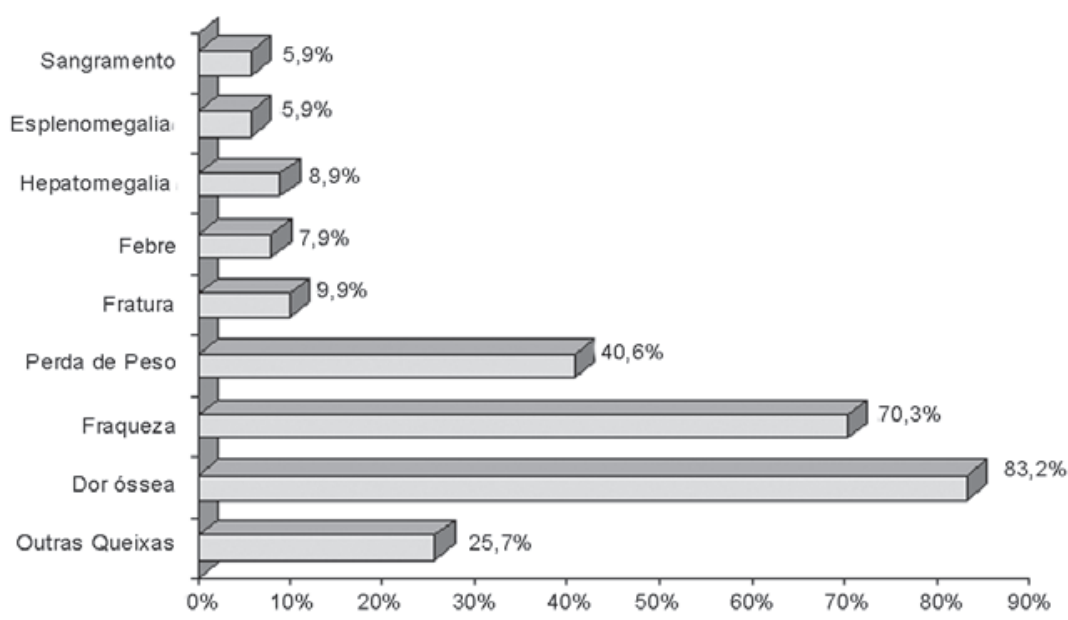

Figura 1. Distribuição de frequência das manifestações clínicas apresentadas pelos pacientes portadores de mieloma múltiplo, ao diagnóstico

dos mostram maior prevalência em brancos, o que justifica a importância de características inerentes da população estudada. ${ }^{2,12}$ Estudo multicêntrico realizado no Brasil ${ }^{9}$ mostrou 80,5\% de prevalência da doença em brancos ou mulatos.

\section{Manifestações clínicas}

Dor óssea foi encontrada em 84 (83,2\%) pacientes. Este resultado é esperado, pois a principal manifestação clínica do MM está relacionada à destruição óssea. ${ }^{15}$ Fratura patológica foi encontrada em 10 (9,9\%) casos. No estudo de Kyle et al., ${ }^{2} 68 \%$ dos pacientes apresentavam dor óssea. $\mathrm{O}$ fato da manifestação clínica mais frequente, ao diagnóstico, ser dor óssea, confirma doença em plena atividade e corrobora para o achado de estádio clínico mais avançado da doença, observado nos pacientes da América do Sul. ${ }^{9}$ Fraqueza foi demonstrada em 71 (70,3\%) e perda de peso em 41 (40,6\%) pacientes. A fraqueza é um sintoma comum no MM e, segundo Kyle, ${ }^{16}$ está presente em dois terços dos pacientes ao diagnóstico. A perda de peso encontrada foi maior que a demonstrada na literatura (24\%), ${ }^{2}$ o que pode refletir possível estádio avançado da doença. Febre foi documentada em oito $(7,9 \%)$ pacientes, podendo estar associada a processos infecciosos não diagnosticados ou a estádio avançado. Hepatomegalia e esplenomegalia foram encontradas em nove $(8,9 \%)$ e seis $(5,9 \%)$ pacientes, respectivamente, como mostra a Figura 1.

\section{Resultados laboratoriais}

A hemoglobina variou de 4,8 g/dL a 16,5 g/dL, com média de 9,5 g/dL ( $\mathrm{DP}=2,4)$. Dos pacientes estudados, 82 $(81,2 \%)$ apresentavam dosagem de hemoglobina inferior a $12,0 \mathrm{~g} / \mathrm{dL}$. Estudo de Kyle et al. ${ }^{2}$ evidenciou anemia em $62 \%$ dos casos, ao diagnóstico. Apenas seis (5,9\%) pacientes apresentavam trombocitopenia (plaquetas $<100.000 / \mathrm{mm}^{3}$ ) ao diagnóstico, semelhante à frequência observada por Kyle et $a l .,{ }^{2}$ em que 5\% dos pacientes apresentavam-se plaquetopênicos. A creatinina variou de $0,5 \mathrm{mg} / \mathrm{dL}$ a $18,2 \mathrm{mg} / \mathrm{dL}$ (média de 2,1 e DP= 2,5). Insuficiência renal é comum no $\mathrm{MM}$, valores elevados de creatinina sérica ( $\geq 2 \mathrm{mg} / \mathrm{dL}$ ) foram encontrados em 24 (23,8\%) pacientes avaliados. Kyle et $a .^{2}$ mostraram que quase um terço dos pacientes apresentavam creatinina $>2 \mathrm{mg} / \mathrm{dL}$ ao diagnóstico.

Uma importante causa de comprometimento renal no MM é a hipercalcemia. A dosagem de cálcio sérico variou de 5,0 mg/dL a 15,1 mg/dL, média de 9,8 mg/dL e DP=1,6. A hipercalcemia (cálcio sérico $=10,5 \mathrm{mg} / \mathrm{dL}$ ) estava presente em $24(23,8 \%)$ dos pacientes estudados. O valor obtido foi semelhante ao encontrado na literatura, onde a hipercalcemia é documentada entre $15 \%$ a $30 \%$ dos casos. ${ }^{1,2}$ Outra importante causa de comprometimento renal é a hiperuricemia, a dosagem de ácido úrico variou de 3,1 mg/dL a 15,1 mg/dL, apresentando média de 7,0 mg/dL (DP=2,5), sendo maior que $8 \mathrm{mg} / \mathrm{dL}$ em 25 (24,8\%) pacientes. A Tabela 1 mostra a média e desvio padrão dos principais exames laboratoriais envolvidos na avaliação inicial do paciente com MM.

Na eletroforese de proteínas séricas, a fração gamaglobulina variou de 0,3 g/dL a 9,4g/dL (média de 3,45 g/dL), enquanto a fração das betaglobulinas variou de $0,1 \mathrm{~g} / \mathrm{dL}$ a

Tabela 1. Resultados dos exames laboratoriais dos pacientes portadores de mieloma múltiplo

\begin{tabular}{lccccc}
\hline & $\mathrm{N}$ & Mínimo & Máximo & Média & $\begin{array}{c}\text { Desvio } \\
\text { Padrão }\end{array}$ \\
\hline Hemoglobina & 99 & 4,8 & 16,5 & 9,5 & 2,4 \\
Plaquetas & 100 & 40,0 & 715,0 & 249,0 & 119,0 \\
Creatinina & 101 & 0,5 & 18,2 & 2,1 & 2,5 \\
Cálcio sérico & 92 & 5,0 & 15,1 & 9,8 & 1,6 \\
Acido úrico & 75 & 3,1 & 15,1 & 7,0 & 2,5 \\
Desidrogenase & 71 & 86,0 & 1531,0 & 448,6 & 218,1 \\
$\quad$ lática (LDH) & 99 & 1,7 & 5,9 & 3,5 & 0,8 \\
Albumina & 99 & 1,4 & 14,5 & 4,6 & 2,5 \\
\hline B2 microglobulina & 74 & 1,4 & & &
\end{tabular}


Tabela 2. Presença de pico monoclonal à eletroforese de proteínas séricas dos 81 pacientes portadores de mieloma múltiplo

\begin{tabular}{lcc}
\hline \multicolumn{1}{c}{ Localização } & Presença de pico monoclonal \\
\hline Pico monoclonal & 53 & $\%$ \\
Pico monoclonal & 12 & 65,9 \\
Pico biclonal & 1 & 14,1 \\
Ausência de pico & 16 & 1,2 \\
\hline Total & 85 & 18,8 \\
\hline
\end{tabular}

Tabela 3. Distribuição dos pacientes portadores de mieloma múltiplo de acordo com o tipo de imunoglobulina monoclonal

\begin{tabular}{lcc}
\hline \multicolumn{1}{c}{ Tipo de proteina M } & N & $\%$ \\
\hline $\operatorname{lgG}$ & 48 & 57,8 \\
$\lg A$ & 14 & 16,9 \\
Cadeia leve & 19 & 22,9 \\
IgG/lgE & 1 & 1,2 \\
Não secretor & 1 & 1,2 \\
\hline Total & 83 & 100 \\
\hline
\end{tabular}

Tabela 4. Frequência das lesões ósseas encontradas em pacientes portadores de mieloma múltiplo, ao diagnóstico, de acordo com a graduação utilizada por Dürie \& Salmon (1975) à radiografia convencional

\begin{tabular}{lcc}
\hline Grau de compretimento ósseo & $\mathrm{N}$ & $\%$ \\
\hline Grau 0 - Osso normal & 16 & 16,2 \\
Grau 1 - Osteoporose & 11 & 11,1 \\
Grau 2 - Lesões osteolíticas & 47 & 47,4 \\
$\begin{array}{l}\text { Grau 3 - Destruição } \\
\text { esquelética extensa e }\end{array}$ & 25 & 25,3 \\
Fraturas maiores & & \\
\hline Total & 99 & 100,0 \\
\hline
\end{tabular}

7,3 g/dL (média de 1,07g/dL). Entre os pacientes analisados, 56 (65,9\%) apresentaram pico monoclonal na região de gamaglobulinas, 12 (14,1\%) em betaglobulinas, um (1,2\%) apresentou pico biclonal e 16 (18,8\%) não apresentaram pico monoclonal sérico, conforme observado na Tabela 2. Resultado semelhante ao obtido por Kyle, ${ }^{16}$ onde o pico das gamaglobulinas estava presente em $53 \%$ e das betaglobulinas em $21 \%$.

Conforme a Tabela 3, o MM tipo IgG foi o mais frequente ( $57,8 \%)$, seguido pelo tipo cadeia leve (22,9\%), conforme descrito na literatura. ${ }^{2}$

\section{Estudo radiológico do esqueleto}

Radiografias convencionais de esqueleto mostraram alterações em 83 (83,8\%) dos casos. O comprometimento mais observado foi presença de lesões osteolíticas $(47,4 \%)$, que são características dessa neoplasia (Tabela 4). Estudos radiográficos convencionais são universalmente utilizados na avaliação inicial do paciente com MM e apresentam altera- ções em 75\% dos pacientes que consistem de lesões osteolíticas em saca-bocado, osteoporose ou fraturas. ${ }^{15}$

Estudo de sobrevida

Ao final do estudo, em março/2007, havia 70 (69,4\%) pacientes vivos ou censorados e 31 (30,6\%) pacientes mortos. A sobrevida global dos 101 pacientes analisados foi de 66,52 meses, variando de 1 mês a 111 meses (FIG.6). A mediana do tempo de seguimento foi de 20 meses. A densidade de incidência da mortalidade, obtida pela divisão do número de óbitos pelo índice pessoa tempo, foi de 0,01 (31/2655). A sobrevida do paciente com MM varia de poucos meses a mais de dez anos, com sobrevida média aproximada de três a quatro anos. ${ }^{18}$

Na análise univariada, pacientes com creatinina menor que $2 \mathrm{mg} / \mathrm{dL}$ apresentaram sobrevida média de 73,08 meses, enquanto aqueles com creatinina maior ou igual a $2 \mathrm{mg} / \mathrm{dL}$ tiveram sobrevida média de 56,52 meses. A diferença de sobrevida foi significante $(\mathrm{p}=0,0067)$. Em relação à hemoglobina, pacientes com hemoglobina maior ou igual a 8,5g/dL apresentaram sobrevida maior (80,36 meses) quando comparados àqueles com hemoglobina menor que 8,5g/dL (55,56 meses), p=0,0014. Ao considerarmos o estadiamento clínico de Durie \& Salmon, obtivemos a maioria dos pacientes estudados, 63 (62,4\%), em estádio III, evidenciando doença avançada e pior prognóstico. No estádio II encontramos $32(31,7 \%)$ pacientes, enquanto apenas $6(5,9 \%)$ pacientes encontravam-se no estádio I. Ao analisarmos a sobrevida em nossa casuística, obtivemos média de 83,33 meses para o estádio I, 81,25 meses para estádio II e 61,90 meses para estádio III. A diferença de sobrevida entre os três grupos foi significante $(\mathrm{p}=0,009)$. Estudo Sul Americano realizado por Hungria et al., ${ }^{9}$ também mostrou que a maioria dos pacientes portadores de MM está em estádio avançado, no momento do diagnóstico: 60 (8\%) estádio I, 91 (12\%) estádio II e 600 (80\%) no estádio III.

Enquanto, em nosso meio, a maior parte dos pacientes apresenta-se em estádio III ao diagnóstico, essa faixa, na literatura internacional, encontra-se abaixo de $55 \% .^{16,19,20}$

Em relação ao ISS, a maior parte dos pacientes (31) encontrava-se no estádio II (42,5\%), 22 (30,1\%), no estádio III, e $20(27,4 \%)$ no estádio I. Vinte e oito pacientes não possuiam dados suficientes no prontuário para a classificação. Greipp et al. ${ }^{7}$ obtiveram uma distribuição parecida: 3.106 (28,9\%) dos pacientes no estádio I; 4.032 (37,5\%) no estádio II e 3.612 (33,6\%) no estádio III. Em um estudo brasileiro ${ }^{21}$ aplicaram o ISS e obtiveram resultados semelhantes: estádio I - 23 (13,4\%); estádio II - 73 (42,4\%) e estádio III- 76 (49,2\%). Ao analisarmos a sobrevida, obtivemos média de 95,0 meses para o estádio I, 63,64 meses para estádio II e 54,84 meses para estádio III. A diferença de sobrevida entre os três grupos foi significante $(p=0,01)$. A sobrevida encontrada por Greipp et al. ${ }^{7}$ foi um pouco menor, com pacientes no estádio I apresentando sobrevida de 62 meses, estádio II, de 44 
A

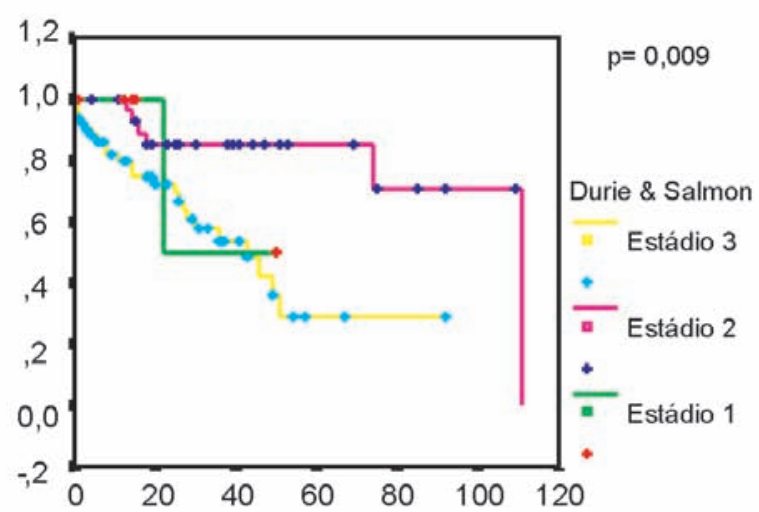

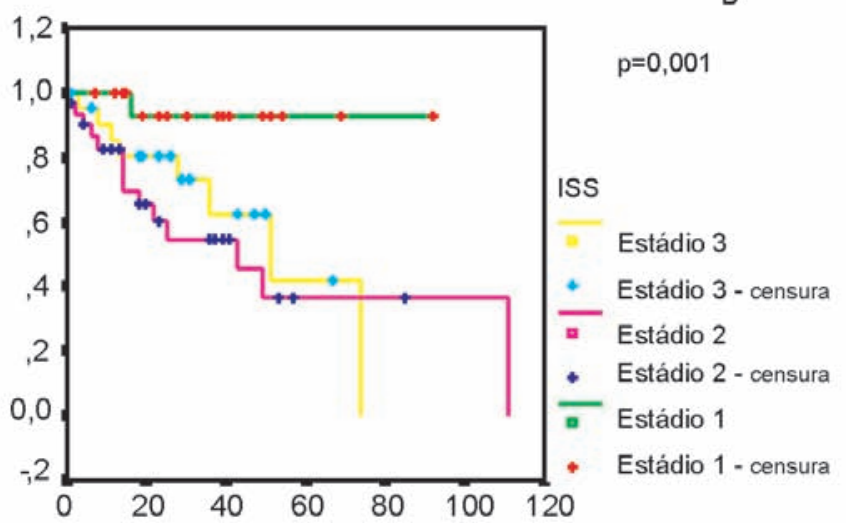

Figura 2. Análise de sobrevida dos pacientes portadores de Mieloma Múltiplo de acordo com o Sistema de Estadiamento Clínico proposto por Durie \& Salmon (A) e Sistema Internacional de Estadiamento (ISS) (B).

Tabela 5. Resultados do estudo de sobrevida, através de análise univariada (Kaplan \& Meier, 1958), em pacientes portadores de mieloma múltiplo

\begin{tabular}{|c|c|c|c|}
\hline Variáveis & Discriminação & $\begin{array}{l}\text { Sobrevida média } \\
\text { (meses) }\end{array}$ & $p$ \\
\hline Perda de peso & $\begin{array}{l}\text { Presença } \\
\text { Ausência }\end{array}$ & $\begin{array}{l}68,29 \\
70,00\end{array}$ & 0,607 \\
\hline Fraqueza & $\begin{array}{l}\text { Presença } \\
\text { Ausência }\end{array}$ & $\begin{array}{l}66,20 \\
76,67\end{array}$ & 0,757 \\
\hline Dor óssea & $\begin{array}{l}\text { Presença } \\
\text { Ausência }\end{array}$ & $\begin{array}{l}69,05 \\
70,59\end{array}$ & 0,910 \\
\hline Co-morbidades & $\begin{array}{l}\text { Presença } \\
\text { Ausência }\end{array}$ & $\begin{array}{l}82,61 \\
65,38\end{array}$ & 0,377 \\
\hline Lesões ósseas & $\begin{array}{l}\text { Presença } \\
\text { Ausência }\end{array}$ & $\begin{array}{l}62,50 \\
69,88\end{array}$ & 0,447 \\
\hline Hemoglobina & $\begin{array}{l}\leq 8,5 \mathrm{~g} \% \\
>8,5 \mathrm{~g} \%\end{array}$ & $\begin{array}{l}55,56 \\
80,36\end{array}$ & 0,001 \\
\hline Plaquetas & $\begin{array}{l}\leq 100.000 / \mathrm{mm}^{3} \\
>100.000 / \mathrm{mm}^{3}\end{array}$ & $\begin{array}{l}57,14 \\
70,21\end{array}$ & 0,163 \\
\hline Creatinina & $\begin{array}{l}<2 \mathrm{mg} / \mathrm{dl} \\
\geq 2 \mathrm{mg} / \mathrm{dl}\end{array}$ & $\begin{array}{l}73,08 \\
56,52\end{array}$ & 0,006 \\
\hline Cálcio & $\begin{array}{l}<10,5 \mathrm{mg} / \mathrm{dl} \\
\geq 10,5 \mathrm{mg} / \mathrm{dl}\end{array}$ & $\begin{array}{l}69,86 \\
57,89\end{array}$ & 0,864 \\
\hline $\begin{array}{l}\text { Desidrogenase } \\
\text { lática (LDH) }\end{array}$ & $\begin{array}{l}\leq 500 \\
>500\end{array}$ & $\begin{array}{l}71,15 \\
73,68\end{array}$ & 0,579 \\
\hline Ácido úrico & $\begin{array}{l}\leq 7,5 \\
>7,5\end{array}$ & $\begin{array}{l}73,91 \\
68,97\end{array}$ & 0,501 \\
\hline $\begin{array}{l}\text { \% Infiltração } \\
\text { plasmocitária }\end{array}$ & $\begin{array}{l}\leq 30 \% \\
>30 \%\end{array}$ & $\begin{array}{l}72,92 \\
63,41\end{array}$ & 0,802 \\
\hline Durie \& Salmon & $\begin{array}{l}\text { Estádio I } \\
\text { Estádio II } \\
\text { Estádio III }\end{array}$ & $\begin{array}{l}83,33 \\
81,25 \\
61,90\end{array}$ & 0,009 \\
\hline ISS & $\begin{array}{l}\text { Estádio I } \\
\text { Estádio II } \\
\text { Estádio III }\end{array}$ & $\begin{array}{l}95,00 \\
63,64 \\
54,84\end{array}$ & 0,014 \\
\hline
\end{tabular}

meses, e estádio III, de 29 meses. Estudo brasileiro ${ }^{9}$ também apresentou menor sobrevida, em especial para o estádio III: estádio I- 53 meses, estádio II- 43 meses e estádio III-23 meses. Todavia, tanto nos trabalhos citados acima quanto no presente estudo, a maioria dos pacientes apresentou doença avançada ao diagnóstico. A Figura 2 mostra as curvas de sobrevida de acordo com os dois sistemas de estadiamento aplicados.

Outros parâmetros, clínicos e laboratoriais foram estudados, porém não apresentaram correlação prognóstica (Tabela 5). Na análise multivariada, apenas a hemoglobina apresentou relação com a sobrevida $(\mathrm{p}=0,012)$. A anemia é uma complicação comum em pacientes com MM e ocorre em dois terços dos pacientes ao diagnóstico. ${ }^{22} \mathrm{~A}$ hemoglobina menor que $8,5 \mathrm{~g} / \mathrm{dL}$ já localiza o paciente em estádio avançado da doença, de acordo com Durie \& Salmon. ${ }^{17}$

\section{Conclusão}

A maioria dos pacientes avaliados apresentava-se em estádio clínico avançado ao diagnóstico, com importante repercussão na sobrevida. A elaboração de projetos de educação continuada para os profissionais de saúde sobre as manifestações clínicas e laboratoriais do MM, principalmente à população médica que atua na Atenção Primária à Saúde no sistema público do Brasil pode auxiliar o diagnóstico precoce dos pacientes portadores de MM e garantir o encaminhamento adequado dos mesmos na rede de atenção à saúde.

\section{Abstract}

Multiple myeloma (MM) is characterized by plasmocyte expansion in the bone marrow and the production of monoclonal immunoglobulin, causing bone destruction, renal failure, hematopoietic suppression and infections. Identification of clinical and laboratory factors in the diagnosis are important to predict survival. The Dürie/Salmon staging system, used for the disease, is based on the correlation of clinical and laboratory parameters on tumoral mass. Studies of the combination of $\beta 2$ microglobulin and albumin resulted in a simple staging system, known as the International Staging System (ISS), which is currently being used. The objectives of this work were to analyze clinical and laboratory 
characteristics in the diagnosis of MM patients and a study of survival. A non-competitive cohort study was performed of 101 MM patients attended in the Hematology Service/Hospital das Clínicas-UFMG who were diagnosed in the period of April 1994 to October 2006.A descriptive analysis of the characteristics at diagnosis and a study of survival were made. The descriptive analysis was achieved using the Kaplan-Meier technique and the Log-Rank test utilized for a comparison of survival curves. The Cox regression test was used for multivariate analysis. The average age of the patients was 63 years, $47.5 \%$ were men and 52.5\% women, with $50.6 \%$ being white, 33.3\% black and 16.1\% mulattos. The most common clinical manifestations were: bone pain (83.2\%) and weakness (70.3\%). Radiographies of the skeleton showed alterations in $83.8 \%$ of the cases. In respect to the Dürie/Salmon staging system, $63(62.4 \%)$ patients were in stage III, 32 (31.7\%) in stage II and 6 (5.9\%) in stage I. Using the ISS classification, 22 (30.1\%) patients were in stage III, 31 (42.5\%) in stage II and 20 (27.4\%) in stage I. Overall survival was 66.52 months with a follow-up of 20 months. In the univariate analysis, creatinine $(p=0.006)$, hemoglobin $(p=0.001)$, the Dürie/Salmon staging system $(p=0.009)$ and ISS $(p=0.014)$ were significant. In the multivariate analysis only hemoglobin ( $p=0.012$ ) was correlated to survival. Most of the patients presented in the advanced stage at diagnosis with repercussions on survival. Rev. Bras. Hematol. Hemoter. 2009; 31(2):63-68.

Key words: Multiple myeloma; prognostic; clinical and laboratory characteristics.

\section{Referências Bibliográficas}

1. Mangan P. Recognizing multiple myeloma. Nurse Pract. 2005; 30(3):14-27.

2. Kyle RA, Gertz MA, Witzig TE, Lust JA, Lacy MQ, Dispenzieri A, et al. Review of 1027 patients with newly diagnosed multiple myeloma. Mayo Clin Proc. 2003;78(1):21-33.

3. Anderson J. Multiple-choice questions revised. Med Teach. 2004; 26(2):110-3.

4. Sirohi B, Powles R. Multiple myeloma. Lancet. 2004; 363 (9412):875-87.

5. Hussein MA, Juturi JV, Lieberman I. Multiple myeloma: present and future. Curr Opin Oncol. 2002;14(1):31-5.

6. Hideshima T, Bergsagel PL, Kuehl WM, Anderson KC. Advances in biology of multiple myeloma: clinical applications. Blood. 2004;104(3):607-18.

7. Greipp PR, San Miguel J, Durie BG, Crowley JJ, Barlogie B, Bladé $\mathrm{J}$, et al. International staging system for multiple myeloma. J Clin Oncol. 2005;23(15):3412-20.

8. Zaidi AA, Vesole DH. Multiple myeloma: an old disease with new hope for the future. CA Cancer J Clin. 2001;51(5):273-85.

9. Hungria VT, et al. South American Multiple Myeloma Study: Epidemiological and clinical characteristics of 751 patients. Haematologica. 2005a;90 (s1):abstract 521.

10. Kaplan EL, Meier P. Nonparametric estimation from incomplete observations. J Am Stat Assoc. 1958;53:457-81.

11. Cox DR. Regression models and life tables. J R Stat Soc. 1972; 34: 187-220.

12. Faria RMD, et al. Prognostic value of clinical, laboratory and morphological characteristics in Multiple Myeloma. Cancer Res Ther Cont. 1999;10:155-161.
13. Bouchardy C, Mirra AP, Khlat M, Parkin DM, de Souza JM, Gotlieb SL. Ethnicity and cancer risk in São Paulo, Brazil. Cancer Epidemiol Biomarkers Prev. 1991;1(1):21-7.

14. Benjamin M, Reddy S, Brawley OW. Myeloma and race: a review of the literature. Cancer Metastasis Rev. 2003;22(1):87-93.

15. Hungria VTM. Doença óssea em Mieloma Múltiplo. Rev. Bras. Hematol. Hemoter. 2007;29(1):60-6.

16. Kyle RA. Multiple myeloma: review of 869 cases. Mayo Clin Proc. 1975;50(1):29-39.

17. Durie BG, Salmon SE. A clinical staging system for multiple myeloma. Cancer. 1975;36(3):842-54.

18. Samson D, Singer C. CME Haematology-1. Clin Med. 2001; 1 (5): 365-70.

19. Turesson I, Abildgaard N, Ahlgren T, Dahl I, Holmberg E, Hjorth $\mathrm{M}$, et al. Prognostic evaluation in multiple myeloma: an analysis of the impact of new prognostic factors. Br J Haematol. 1999; 106(4):1005-12.

20. Knudsen LM, Hjorth M, Hippe E. Renal failure in multiple myeloma: reversibility and impact on the prognosis. Nordic Myeloma Study Group. Eur J Haematol. 2000;65(3):175-81.

21. Hungria VT. Validation of Internacional Staging System for multiple myeloma: a retrospective analysis of 172 patients at two brazilian centers. Haematologica. 2005b;90 (s1): abstract 312.

22. Cançado RD. Mieloma múltiplo e anemia. Rev. Bras. Hematol. Hemoter. 2007;29(10):67-76.

Avaliação: Editor e dois revisores externos Conflito de interesse: não declarado

Recebido: 17/09/2008

Aceito: 28/12/2008 